\section{Case Reports in Nephrology and Dialysis}

\title{
Rituximab Treatment in a Patient with Kimura Disease and Membranous Nephropathy: Case Report
}

\author{
Roald Vissing-Uhre $^{a}$ Alastair Hansen ${ }^{b}$ Susanne Frevert ${ }^{c}$ Ditte Hansen ${ }^{d}$ \\ aDepartment of Pulmonology and Infectious Medicine, Nordsjaellands Hospital, Hillerod, \\ Denmark; ' Department of Pathology, Herlev Hospital, Herlev, Denmark; 'Department of \\ Diagnostic Radiology, Rigshospitalet, Copenhagen, Denmark; 'Department of Nephrology, \\ Herlev Hospital, Herlev, Denmark
}

\section{Keywords}

Glucocorticoid · Nephrotic syndrome · Glomerular disease

\begin{abstract}
Kimura disease (KD) is a chronic, inflammatory disorder with slowly developing subcutaneous tumor-like swellings, often occurring in the head and neck region. KD is diagnosed based on histology, elevated levels of immunoglobulin type $E$, and increased peripheral eosinophil granulocytes. KD may coexist with glomerular renal diseases, and this case report is based on a patient with KD-associated membranous nephropathy. Patients with membranous nephropathy without KD have demonstrated responsiveness to treatment with monoclonal anti-CD20 antibodies. This case report is the first to investigate the effect of rituximab treatment in a patient with KD-associated membranous nephropathy. A 30-year-old Italian man living in Denmark was diagnosed with Kimura's disease based on subcutaneous nodules with eosinophil angiolymphoid hyperplasia. The patient was admitted to the hospital due to nephrotic syndrome. Serology showed eosinophil granulocytosis and negative PLA2-receptor antibody. Renal biopsy showed membranous nephropathy, and the patient was treated with systemic methylprednisolone followed by cyclosporin and then cyclophosphamide with only partial remission. Ultimately, treatment with intravenous rituximab was initiated, which resulted in overall remission and no nephrotic relapses at 30 months of follow-up. Thus, intravenous rituximab effectively decreased proteinuria and prevented nephrotic relapses in a patient with treatment-refractory membranous nephropathy due to KD.
\end{abstract}

(C) 2021 The Author(s).

Published by S. Karger AG, Basel

\section{Karger $\stackrel{2}{\circ}$}




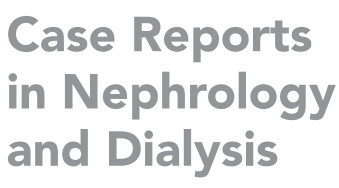

Introduction

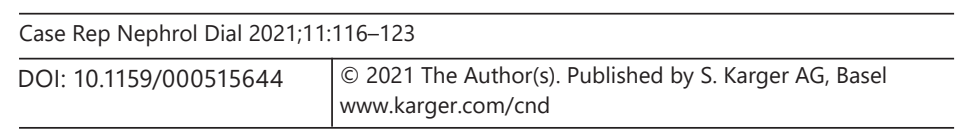

Vissing-Uhre et al.: Rituximab Treatment in Kimura's Disease

Kimura disease (KD) is a chronic, inflammatory disorder with slowly developing painless, subcutaneous tumor-like swellings that histologically consist of vascular endothelium hyperplasia and lymphoid follicles with presence of eosinophils. The tumor-like swellings typically occur in the head and neck region, and clinical examination may also reveal solitary or multiple nontender lymph nodes in the cervical region. KD is a rare disease, and the global prevalence is estimated to be 300 patients with the highest incidence occurring in East Asian men aged 20-40 years [1].

Renal manifestations coexist in $10-60 \%$ of the patients, and $12-16 \%$ of the patients display nephrotic syndrome characterized by clinical proteinuria ( $>3.5 \mathrm{~g} /$ day) [2]. KD is diagnosed by histopathological examination of a surgical biopsy in conjunction with elevated levels of serum immunoglobulin type $\mathrm{E}$ and increased peripheral blood eosinophilia [3]. KD may be associated with different kidney diseases such as mesangial proliferative GN, minimal change disease, IgA nephropathy, membranous nephropathy (MN), focal glomerular sclerosis, or acute tubular injury [1]. Skin lesions are often the first symptom of the disease, and coexisting renal manifestations are typically evident months later.

As in isolated cases of glomerular diseases, patients with KD and glomerular diseases are responsive to immunosuppressive treatment protocols, including corticosteroid, cyclosporin, and cyclophosphamide [4-6]. Still, frequent nephrotic relapses and lack of remission in proteinuria enhance the risk of developing end-stage renal disease [7].

No reports describe the effect of rituximab in patients with KD associated with MN. Here, we present a case report of the effect of rituximab treatment in a patient with KD and MN.

\section{Case Presentation}

We report a 30-year-old Italian man living in Denmark, who in 1998 presented with multiple tumor-like subcutaneous swellings as the first manifestations of KD. The swellings were located on the medial and lateral part of the right scapula and left trapezius muscle. Histological examination of a skin biopsy from the left scapula showed eosinophil angiolymphoid hyperplasia. Surgical excision of the tumor near the right scapula excluded underlying malignancy, and no further medical treatment was undertaken at that time.

\section{Clinical Features and Laboratory Findings}

In late 2014, only the previously removed tumor on the lateral part of the right scapula reoccurred. Compared to imaging performed before the surgical excision, ultrasound and MRI showed reoccurrence and progression of tumor size. The round, heterogeneous tumor was $3 \times 5 \times 7 \mathrm{~cm}$ and infiltrated the paravertebral musculature with diffuse contrast enhancement as shown in Figure 1. MRI revealed vascular proliferation in relation to the tumor with one lymph node appearing with an arteriovenous malformation contained of shunted blood. Full thickness biopsy of the tumor demonstrated epithelial angiolymphoid hyperplasia with eosinophilia. Thereby, malignancy was excluded again, and no further surgical treatment was undertaken at this point. The subsequent month, the patient was admitted to the hospital with lower extremity, facial and periorbital edemas, and lymphadenopathy of the neck. Biochemical findings showed nephrotic syndrome with a low serumalbumin of $15 \mathrm{~g} / \mathrm{L}$ and severe proteinuria with a maximal excretion of $13.5 \mathrm{~g} /$ day. Hematology demonstrated leukocytosis with elevated levels of eosinophil granulocytes of $4.88 \times$ $10^{9} / \mathrm{L}$ and a blood platelet count of $456 \times 10^{9}$ platelets/L. Blood analysis indicated no complement abnormalities of plasma C3 or C4, and plasma IgG, IgA, and IgM were normal.

\section{Karger'}


Fig. 1. MRI sagittal T2- and T1-weighted fatsaturated contrast-enhanced image showing a well-defined heterogeneous hypervascular process with marked enhancement (white arrows). The process is surrounded by numerous arterial feeders (black arrows).

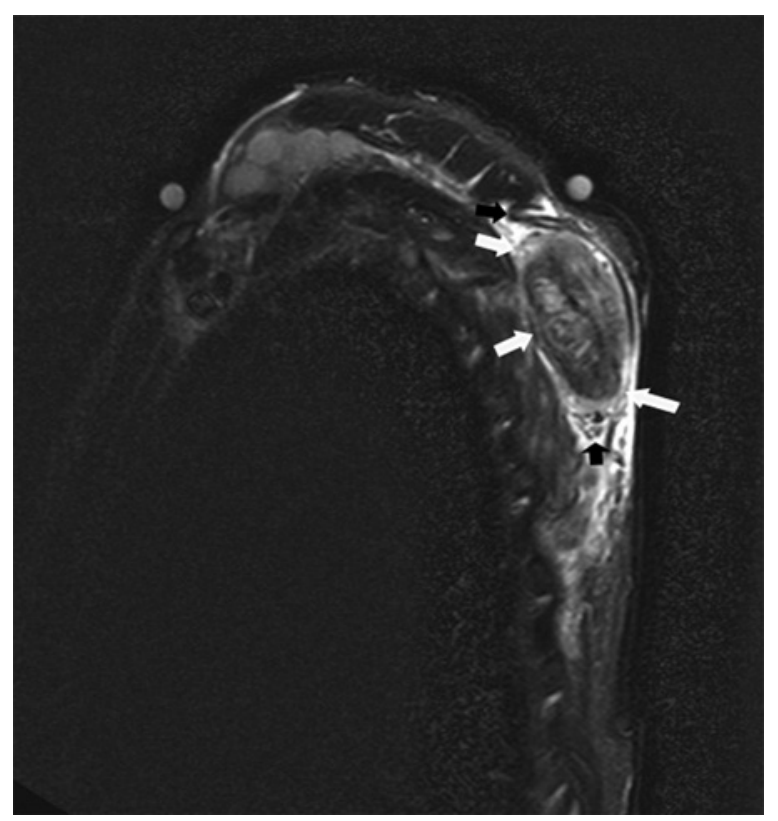

Secondary causes of nephrotic syndrome were excluded by serological tests, demonstrating negative hepatitis serology (types A, B, and C), HIV type 1 + 2, PR3 and MPO-ANCA, anti-GBM, anti-DNA, ANA, and M-component.

Ultrasound of the kidney was normal. Renal biopsy demonstrated diffuse, MN without infiltration of eosinophils in the renal interstitium. Electron microscopy revealed characteristics of MN showing epimembranous electron-dense deposits (Fig. 2d). Immunofluorescence showed granular IgG and complement deposits of C3 and C1Q together with deposition of kappa and lambda in the basal membranes. There was no IgA or IgM deposition. In conclusion, the patient was diagnosed with KD with nephrotic syndrome characterized by MN. PLA2Rantibody was negative. Histological examination of the tumor swelling reconfirmed the presence of eosinophil angiolymphoid hyperplasia, shown in Figure 2a.

\section{Treatment and Follow-Up}

Case reports indicated that KD with associated MN could effectively be treated with prednisolone, resulting in decrease of proteinuria and regression of tumor masses [8]. In accordance with findings of case reports on the effects of prednisolone therapy in patients with KD and nephrotic syndrome, oral prednisolone was initiated at the dose of $50 \mathrm{mg} /$ day to induce remission of nephrotic syndrome (Fig. 3) [4].

Symptomatic treatment with cetirizine $10 \mathrm{mg} /$ day and antihypertensive treatment with enalapril $5 \mathrm{mg} /$ day was initiated. Dose escalation of enalapril was not possible due to orthostatism. During 3 months of treatment with prednisolone, edemas and cervical lymphadenopathy resolved. The size of the scapular tumor persisted, and treatment of the tumor was performed by transcatheter embolization of a feeding branch from the subclavian artery supplying the arteriovenous malformation, resulting in some reduction in tumor mass and pain. Though embolization did improve P-albumin to $30 \mathrm{~g} / \mathrm{L}$, severe proteinuria persisted $(7.1 \mathrm{~g} / \mathrm{L})$, shown in Figure 3. Prednisolone was tapered to $15 \mathrm{mg} /$ day, while introducing cyclosporin, shown in Figure 4. After 3 months of concomitant prednisolone and cyclosporin therapy, partial remission was observed, but proteinuria increased when prednisolone was further tapered. The patient was steroid dependent, and levels of proteinuria indicated that

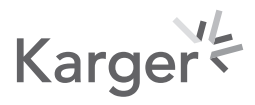




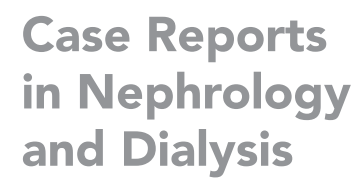

\begin{tabular}{l|l}
\hline \multicolumn{2}{l}{ Case Rep Nephrol Dial 2021;11:116-123 } \\
\hline DOI: 10.1159/000515644 & $\begin{array}{l}\text { (c) 2021 The Author(s). Published by S. Karger AG, Basel } \\
\text { www.karger.com/cnd }\end{array}$ \\
\hline
\end{tabular}

Vissing-Uhre et al.: Rituximab Treatment in Kimura's Disease

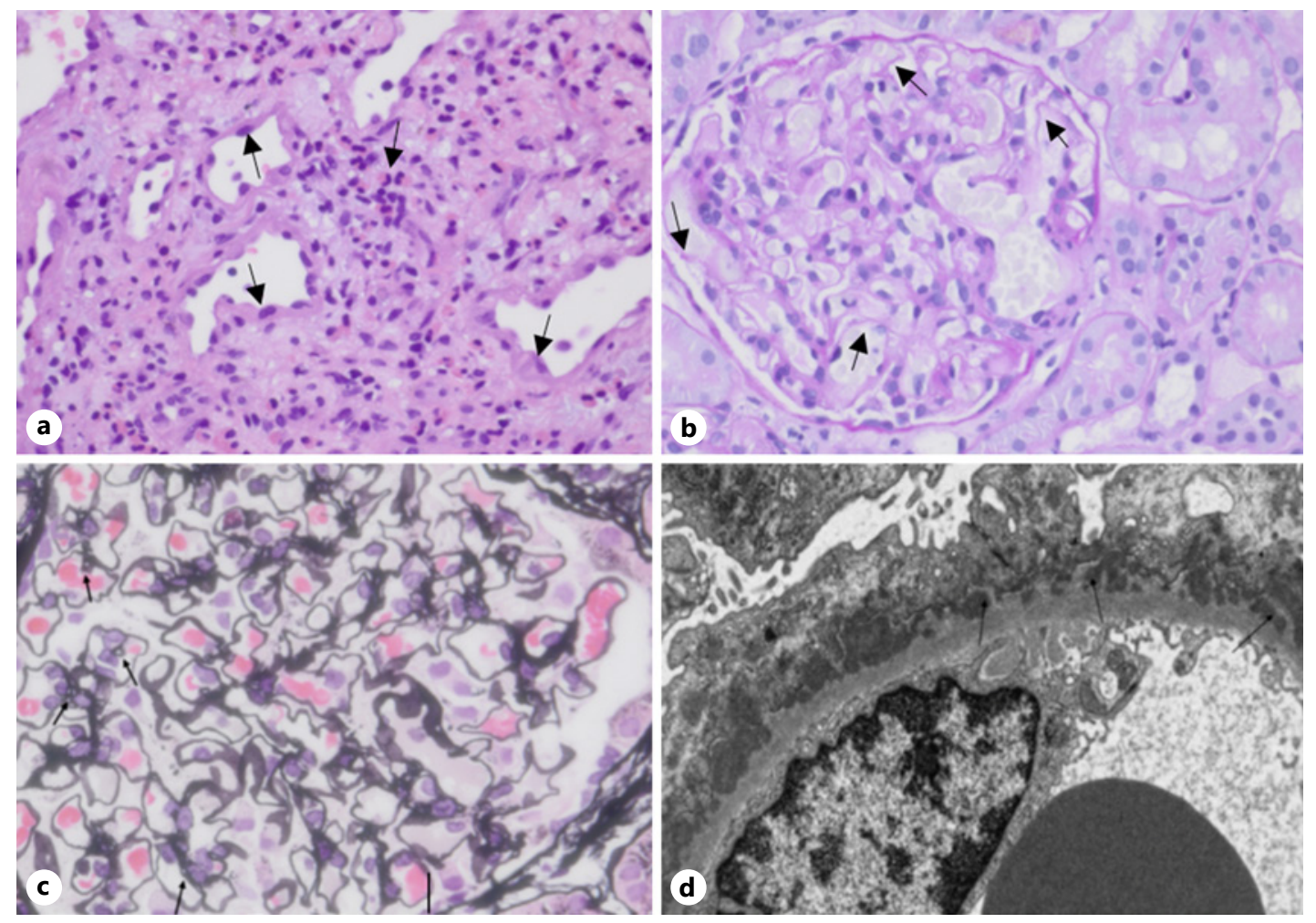

Fig. 2. Histological examination of dermal and renal biopsies. a Dermal biopsy with angiolymphoid hyperplasia with eosinophilia, showing thin-walled vessel lined by plump endothelial cells. The surrounding stroma is infiltrated by lymphocytes and prominent eosinophils (H\&E, ×320). b Glomerulus with early-stage membranous glomerulonephritis. The basement membranes are thin and surrounding potent capillaries. Normal tubules and interstitium (H\&E, ×400). c Glomerulus with early-stage membranes glomerulonephritis showing only rare holes (arrows) in the glomerular basement membranes (methenamine silver PAS, $\times 400$ ). d Electron micrograph of the glomerular capillary wall showing diffuse subendothelial electron-dense deposits. There are focal spikes with lateral extensions (arrows), indicating disease stage II. Spikes were not visible by light microscopy (uranyl acetate and lead citrate, $\times 11,000$ ).

monotherapy with cyclosporin was not possible. Cyclosporin was discontinued, and prednisolone monotherapy in doses of $15 \mathrm{mg} /$ day was maintained and cyclophosphamide 100 $\mathrm{mg} /$ day was added for 6 months. After this treatment, the patient experienced partial remission in proteinuria, normal p-albumin, and eosinophils normalized, and prednisolone was effectively tapered. However, 1 year later, the patient experienced relapse of nephrotic syndrome and increased eosinophilia, while MR showed no further progression of the scapular tumor.

At this time, no studies had evaluated the effect of intravenous application of rituximab on patients with KD and glomerular disease. However, the established literature existed on the effect of rituximab in treating patients with MN without KD. The patient received $1 \mathrm{~g}$ of intravenous rituximab, administered at the Department of Nephrology, as a final treatment strategy, which was given a total of 2 times with 2-week intervals. Rituximab induced remission in nephrosis and normalization of peripheral eosinophilia, which persisted after 30 months of follow-up, and there were no signs of reappearance of cervical lymphadenopathy or clinical progression of the scapular tumor. Treatment was well tolerated, and the patient experienced no side effects. 


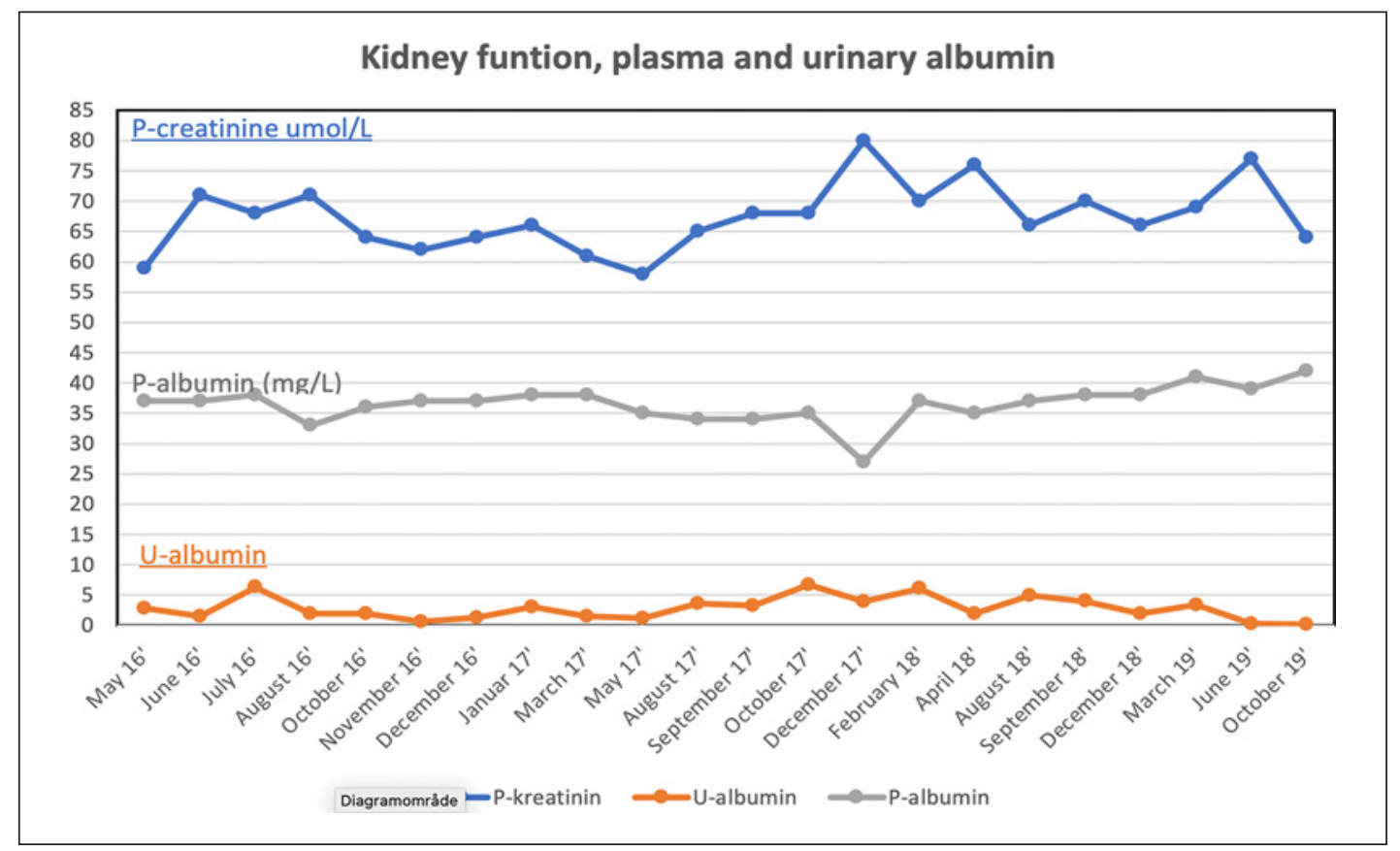

Fig. 3. Serial changes in plasma-creatinine ( $\mu \mathrm{mol} / \mathrm{L})$, p-albumin $(\mathrm{mg} / \mathrm{L})$, and $\mathrm{u}$-albumin $(\mathrm{g} / \mathrm{L})$ observed in the patient. During the treatment period, p-creatinine and eGFR were stable, while p-albumin decreased as the patient became nephrotic before initiation of intravenous rituximab.

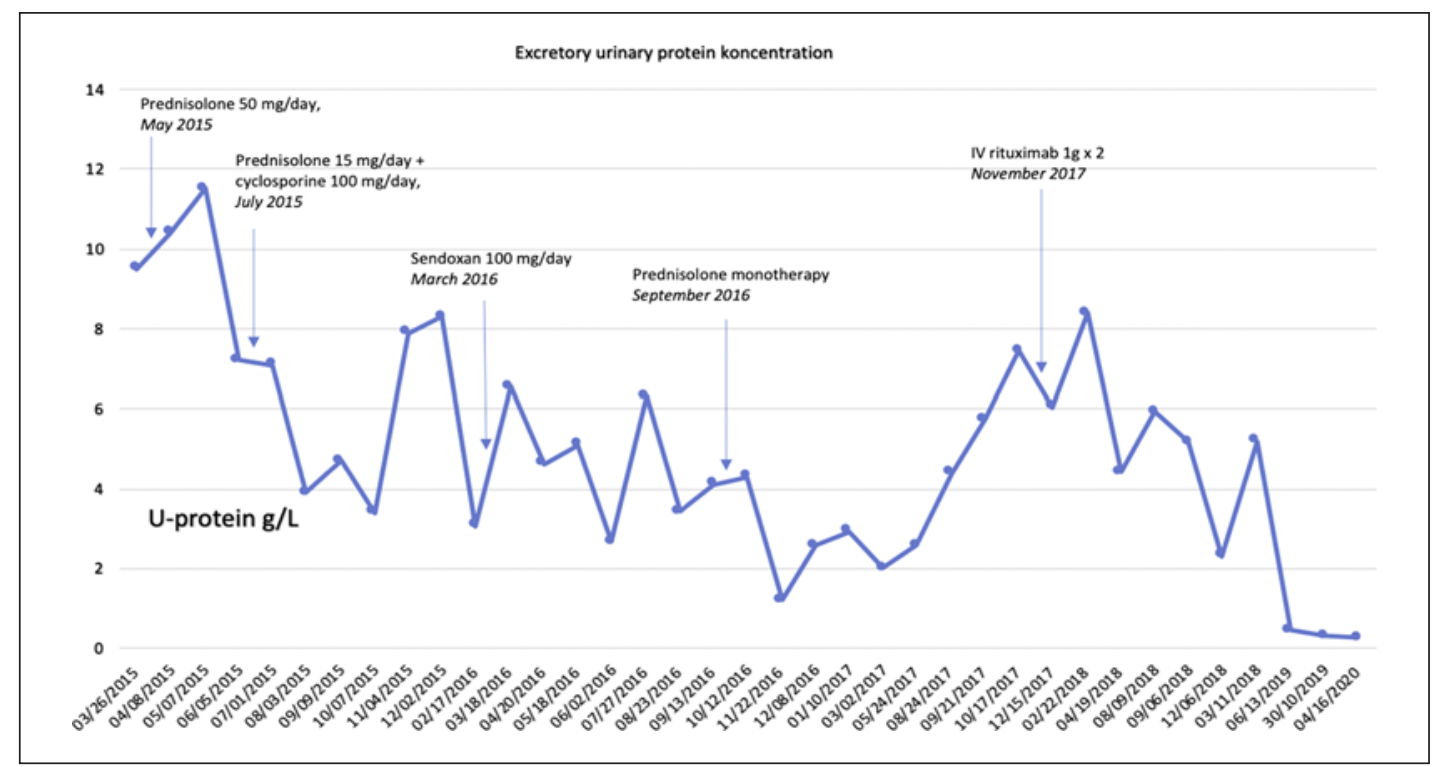

Fig. 4. Serial changes in urinary protein (g/L/day) during 3 months of monotherapy with prednisolone 50 $\mathrm{mg} /$ day; the patient maintained severe proteinuria. Cyclosporin was added, and concomitant prednisolone and cyclosporine therapy induced partial remission. However, tapering of prednisolone induced relapse of severe proteinuria. Cyclosporine was discontinued, and concomitant prednisolone and cyclophosphamide of $100 \mathrm{mg} /$ day was administered for 6 months in which the patient experienced partial remission. One year later, a nephrotic relapse occurred. The patient received 2 doses of intravenous rituximab, which induced total remission which persisted after 30 months of follow-up. 


\section{Discussion/Conclusion}

This case report is the first to describe the effect of intravenous rituximab treatment in a patient with KD and coexisting MN. Treatment was well tolerated, and the patient showed no signs of relapse during 30 months of follow-up.

KD with MN is rare, existing in only $15 \%$ of all patients with $\mathrm{KD}$-associated glomerular diseases [1]. Thus, causalities between KD and MN and the effect of different kinds of treatments are sparsely described. MN is classified as either idiopathic MN with unknown origin or secondary MN caused by systemic diseases such as malignancy, autoimmune, and various infectious diseases. It is considered an immune-mediated glomerular disease with pathological deposits of immune complexes mainly in the subepithelial region of the glomerular basal membrane (GBM) in kidney glomeruli. In the majority of cases of idiopathic MN, the circulating antibody M-type receptor for secretory phospholipase A2 (PLA2R1) expressed on podocytes on GBM plays a role in depositions of immune complexes near GBM [9]. MN with negative PLA2R antibodies may be considered as secondary to other conditions, and the patient was screened for other diseases than KD [10].

In this report, the histological renal manifestations of the patient with KD resembled those typically presented in MN, in line with the findings of Obata et al. [8]. It has been proposed that circulating antibodies planted in subepithelial sites of GBM play a part of the mechanism of forming immune complex deposits with antibodies inducing secondary MN. Immune complexes are formed by antibody-antigen interactions, where the circulating antigens of secondary conditions such as hepatitis B and C and tumors are deposited [11]. Though immune-mediated mechanisms leading to KD and MN seem correlated, the pathogenesis is not yet well defined.

Fouda et al. [4] have previously reported short-term remission of proteinuria in a patient with KD and MN on prednisolone monotherapy. Based on this report, we initiated prednisolone as the first-line treatment in the presented patient, although prednisolone monotherapy in patients with MN is generally not recommended [12]. Prednisolone proved effective in regressing cervical lymphadenopathy, while the size of the scapular tumor remained unchanged. This was surprising, since Obata et al. [8] had previously demonstrated diminishing tumors on prednisolone monotherapy in cases of KD with associated MN. Despite initial remission of the nephrotic syndrome, in line with findings of Gaillard et al. [13], sustained remission on prednisolone monotherapy was not possible, and the patient experienced relapse of proteinuria. The patient followed a natural course of relapsing nephrosis with severe proteinuria as typically seen in cases of $\mathrm{MN}$, and hence it was decided to apply common treatment protocols of MN to the patient. Cyclosporin increases remission in patients with MN, although the risk of relapse is high when withholding treatment [14]. Cyclosporin combined with prednisolone was effective in terms of reduction of proteinuria in our patient but did not allow prednisolone to be tapered. As such, the patient persisted steroid dependent.

Cyclophosphamide has been proven to induce remission and reduce risk of relapse in $\mathrm{MN}$, acting by nonselectively destroying B cells [15]. Cyclophosphamide induced remission in our patient, but the proteinuria increased after 1 year and due to the increased risk of toxicity with increasing doses of cyclophosphamide, repeated cyclophosphamide treatment was avoided [16]. Instead, rituximab was applied and induced complete remission of the proteinuria, which has been maintained for 30 months. Corresponding to this important finding, the recent MENTOR trial on cyclosporine versus intravenous rituximab proved rituximab effectiveness in maintaining long-term proteinuria remission up to 24 months [17].

In this case, sclerotherapy of the tumor masses was performed, while no clinical remission in nephrosis occurred. Surgical tumor excision has been reported to induce full remission of

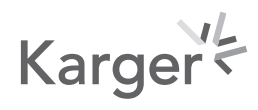


nephrosis in KD with associated MN, suggesting that the angiolymphoid mass might secrete circulating factors that induce glomerular damage [18]. However, further surgical excision at the time of rituximab application was not suggested in our case, since MRI did not demonstrate any tumor progression.

This case report suggests that administration of rituximab brings prolonged benefits up to at least 30 months, greater remission levels, and lack of nephrotic relapse. These findings favor first-line treatment with rituximab, instead of preserving it for treatment-refractory cases. As the remission in proteinuria is a surrogate marker for long-term outcome in these patients with MN, earlier application of rituximab could possibly decrease the risk of endstage renal failure.

\section{Statement of Ethics}

Written informed consent for publication and for accompanying images of this case report was obtained and signed by the patient. Consent is available on request.

\section{Conflict of Interest Statement}

The authors have no conflicts of interest to declare.

\section{Funding Sources}

The authors did not receive any funding.

\section{Author Contributions}

All authors substantially contributed to data acquisition and interpretation. Medical doctor R.V.U. drafted the manuscript. Nephrologist D.H. was responsible for treatment of the patient and a major contributor in critically revising the manuscript. Pathologist A.H. performed histological examination of dermal and renal biopsies. Radiologist S.F. described MR images and was responsible for treating the patient with embolization. All authors read and approved the final manuscript and gave consent to be personally accountable for their contributions.

\section{References}

1 Wang DY, Mao JH, Zhang Y, Gu WZ, Zhao SA, Chen YF, et al. Kimura disease: a case report and review of the Chinese literature. Nephron Clin Pract. 2009;111(1):c55-61.

2 Rajpoot DK, Pahl M, Clark J. Nephrotic syndrome associated with Kimura disease. Pediatr Nephrol. 2000; 14(6):486-8.

3 Abuel-Hajia M, Hurford MT. Kimura disease. Arch Pathol Lab Med. 2007;131(4):650-1.

4 Fouda MA, Gheith O, Refaie A, El-Saeed M, Bakr A, Wafa E, et al. Kimura disease: a case report and review of the literature with a new management protocol. Int J Nephrol. 2010;2010(1):673908-4.

5 Wang YS, Tay YK, Tan E, Poh WT. Treatment of Kimura's disease with cyclosporine. J Dermatolog Treat. 2005; 16(4):242-4.

6 Song R, Xin YL, Wang F, Zhang P, Zhang Y, Wang L, et al. Nephrotic syndrome associated with Kimura's disease: a case report and review of the literature. BMC Nephrol. 2018;19(1):316.

\section{Karger'}


7 Troyanov S, Wall AC, Scholey JW. Idiopathic membraneous nephropathy: definition and relevance of a partial remission. Kidney Int. 2004;66(3):1199-205.

8 Obata Y, Furusu A, Nishino T. Membraneous nephropathy and Kimura's disease manifesting a hip mass. A case report with literature review. Intern Med. 2010;49(14):1405-9.

9 Lai WL, Yeh TH, Chen PM, Chan CK, Chiang WC, Chen YM, et al. Membranous nephropathy: a review on the pathogenesis, diagnosis, and treatment. J Formos Med Assoc. 2015;114:102-11.

10 Dai H, Zhang H, He Y. Diagnostic accuracy of PLA2R autoantibodies and glomerular staining for the differentiation of idiopathic and secondary membranous nephropathy: an updated meta-analysis. Sci Rep. 2015;5: 8803.

11 Liu W, Gao C, Dai H, Zheng Y, Dong Z, Gao Y, et al. Immunological pathogenesis of membranous nephropathy: focus on PLA2R1 and its role. Front Immunol. 2019;10:1809.

12 Cattran DC, Delmore T, Roscoe J, Cole E, Cardella C, Charron R, et al. A randomized controlled trial of prednisone in patients with idiopathic membranous nephropathy. N Engl J Med. 1989;320:210-5.

13 Gaillard J, Rotman S, Girardet C, Spertini F. Successful therapy with mycophenolic acid in a membranous glomerulonephritis due to Kimura disease. Clin Nephrol. 2017;88(4):221-5.

14 Cattran DC, Appel GB, Hebert LA, Hunsicker LG, Pohl MA, Hoy WE, et al. Cyclosporine in patients with steroidresistant membranous nephropathy: a randomized trial. Kidney Int. 2001;59(4):1484-90.

15 Ponticelli C, Altieri P, Scolari F, Passerini P, Roccatello D, Cesana B, et al. A randomized study comparing methylprednisolone plus chlorambucil versus methylprednisolone plus cyclophosphamide in idiopathic membranous nephropathy. J Am Soc Nephrol. 1998;9(3):444-50.

16 Faurschou M, Sorensen IJ, Mellemkjaer L. Malignancies in Wegener's granulomatosis: incidence and relation to cyclophosphamide therapy in a cohort of 293 patients. J Rheumatol. 2007;35(1):100-5.

17 Fervenza FC, Appel GB, Barbour SJ, Rovin BH, Lafayette RA, Aslam N, et al. Rituximab or cyclosporine in the treatment of membranous nephropathy. N Engl J Med. 2019;381:36-46.

18 Lee S, Yi YJ, Ah Jo H, Huh H, Kim KH, Ki Kim D, et al. Remission of secondary membranous nephropathy in a patient with Kimura disease after surgical resection. Kidney Res Clin Pract. 2014;33:157-60. 